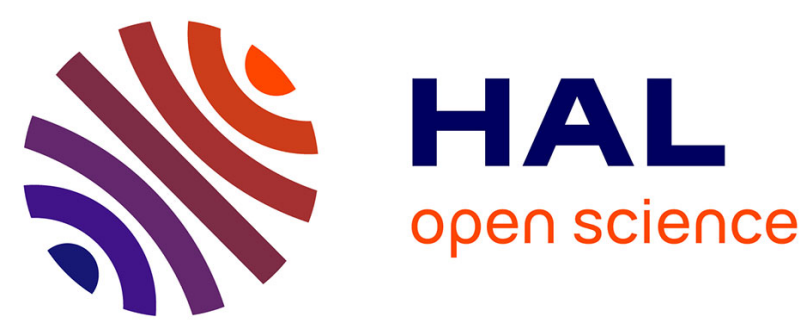

\title{
Bronchoalveolar lavage in systemic sclerosis with lung involvement: role and correlations with functional, radiological and scintigraphic parameters
}

Stefania Volpinari, Renato La Corte, Stefano Bighi, Franco Ravenna, Napoleone Prandini, Andrea Lo Monaco, Francesco Trotta

\section{To cite this version:}

Stefania Volpinari, Renato La Corte, Stefano Bighi, Franco Ravenna, Napoleone Prandini, et al.. Bronchoalveolar lavage in systemic sclerosis with lung involvement: role and correlations with functional, radiological and scintigraphic parameters. Rheumatology International, 2010, 31 (9), pp.1183-1188. 10.1007/s00296-010-1390-9 . hal-00618200

\section{HAL Id: hal-00618200 https://hal.science/hal-00618200}

Submitted on 1 Sep 2011

HAL is a multi-disciplinary open access archive for the deposit and dissemination of scientific research documents, whether they are published or not. The documents may come from teaching and research institutions in France or abroad, or from public or private research centers.
L'archive ouverte pluridisciplinaire HAL, est destinée au dépôt et à la diffusion de documents scientifiques de niveau recherche, publiés ou non, émanant des établissements d'enseignement et de recherche français ou étrangers, des laboratoires publics ou privés. 
Bronchoalveolar lavage in systemic sclerosis with lung involvement: role and correlations with functional, radiological and scintigraphic parameters.

Stefania Volpinari, Renato La Corte, ${ }^{*}$ Stefano Bighi, ${ }^{\circ}$ Franco Ravenna, ${ }^{*}$ Napoleone Prandini, Andrea Lo Monaco, Francesco Trotta.

Rheumatology Section, Department of Clinical and Experimental Medicine-University of Ferrara; * Radiology Service,

${ }^{\circ}$ Interventional Pneumology; ** Nuclear Medicine Department - S. Anna Hospital, Ferrara- Italy.

\section{Corresponding Author:}

Stefania Volpinari, e-mail: svolpinari@email.it.

Department of Clinical and Experimental Medicine, University of Ferrara.

Corso della Giovecca 203, 44100, Ferrara - Italy

(+39) 0532-236314, fax (+39) 0532-207221, (+39) 335-7342906.

KEY-WORDS: Systemic Sclerosis; Interstitial lung disease; alveolitis, bronchoalveolar lavage, pulmonary function tests, lung high resolution computed tomography, alveolar clearance. 


\begin{abstract}
Objectives: To evaluate the role and the prognostic value of bronchoalveolar lavage (BAL) in scleroderma patients with interstitial lung disease.

Methods: We reviewed the records of 79 patients with Systemic Sclerosis (SSc) who had dyspnea and pulmonary involvement and underwent BAL study. Sixty-two patients were prospectively followed-up for 12-36 months and reevaluated by pulmonary function tests (PFTs).
\end{abstract}

Results: 79 SSc patients were enrolled (71 F and 8 M), 55 with limited and 24 with a diffuse form; mean age $55 \pm 13$ years; mean disease duration $55.2 \pm 59$ months. All patients were ANA positive, of these 30 were anti-topoisomerase-1 positive (anti-Topo1) and 22 were anti-centromere positive (ACA).

Thirty-one patients had alveolitis (39.2\%) that was neutrophilic in 12 patients, eosinophilic in 3 and mixed (neutrophilic and eosinophilic) in 16 patients. Compared to patients without alveolitis, those with alveolitis had a significant reduction of carbon monoxide diffusing capacity (DLCO), forced vital capacity (FVC) and more elevated lung high-resolution computed tomography (HRCT) scores. Furthermore, alveolar clearance was significantly accelerated. No differences were found between patients with and without alveolitis regarding disease subsets (diffuse vs limited-SSc); a significant predominance of anti-Topo1 antibodies was found in the alveolitis group and of ACA antibodies in the non alveolitis cohort.

During the follow-up (range:12-36 months) 62 patients, 26 with and 36 without alveolitis were re-evaluated with PFTs. In the alveolitis group, 12 patients (46.1\%) showed stable lung function parameters and 14 had worsened (53.8\%). In this group, 20 patients $(77 \%)$ received cyclophosphamide (CYC): 11 (55\%) worsened (5 of them died of cardiopulmonary complications) and 9 (45\%) remained stable. Six patients couldn't be treated; of these 3 remained stable and 3 worsened.

Among 36 patients with normal BAL, $11(30.5 \%)$ showed stable lung function parameters, 13 improved $(36.1 \%)$ and 12 worsened $(33.3 \%)$; in this last group 2 patients died of extra-pulmonary complications. Six patients, with progression of lung fibrosis, were treated with CYC: 3 of them improved and 3 remained stable.

Conclusions: Our study revealed a trend towards a more severe course in the SSc patients with BAL alveolitis; probably the non significant result is related to the low number of the examined subjects and to the selection criteria. 
However BAL remains the only tool to exclude lung infections and, in our experience, a useful instrument to evaluate interstitial lung disease in SSc patients.

\section{INTRODUCTION}

Lung involvement represents the most relevant complication and the main cause of morbidity and mortality in systemic sclerosis (SSc). During the course of the disease about $80 \%$ of patients with SSc develop alveolar inflammation and pulmonary fibrosis (1). Other than pulmonary function tests (PFTs) and high-resolution computed tomography (HRCT) of the lung, bronchoalveolar lavage (BAL) showed a prognostic value in predicting increased mortality, identifying alveolitis before extensive lung involvement has developed and allowing an earlier intervention (2). Furthermore, BAL cultures and histological lung examination can help to identify patients with infection or other lung complications (3). However, the pathogenetic role of alveolitis detected by BAL in SSc is uncertain because it is not known whether the cells recovered are the cause or a consequence of the lung fibrosis (4).

This study summarises our experience in defining the diagnostic and prognostic value of BAL alveolitis in a series of SSc patients. 


\section{PATIENTS AND METHODS}

In this retrospective study, records of all scleroderma patients with pulmonary interstitial involvement who underwent BAL study were analyzed. All patients fulfilled the criteria proposed by the American College of Rheumatology (5) and were grouped according to LeRoy et al. in patients with limited and diffuse skin involvement (6). Modified Rodnan Skin Score was performed for all patients (7).

The baseline clinical characteristics of the patients, including demographic data, autoantibody status and disease duration (evaluated from the first symptom, except Raynaud's phenomenon), were retrieved from the database. Antinuclear antibodies (ANA) were determined by indirect immunofluorescence using Hep-2 cells as substrates and autoantibody specificities were further assessed by enzyme-linked immunosorbent assay (ELISA) (8).

Inclusion criteria were: a) presence of dyspnea on exertion $\geq$ grade 2 according to the Mahler Baseline Dyspnea index (9); b) carrying out at baseline pulmonary function testing (PFTs), chest HRCT, ${ }^{99 m}$ Technetium-DTPA Lung Scintigraphy with abnormality at least of one of these exams (see below). Current smokers, patients with positive BAL cultures for ongoing infection and patients with pulmonary hypertension (defined as a value of systolic echocardiographic pulmonary pressure $>35 \mathrm{mmHg}$ ) were excluded.

At baseline all patients were on treatment with Ca-antagonist associated in 35 cases with low dosages of Penicillamine (150 or $300 \mathrm{mg} \mathrm{die)}$ and glucocorticoids (methylprednisolone from 4 to $8 \mathrm{mg} / \mathrm{die}$ ) and in 44 cases only with low doses of glucocorticoids.

All patients provided informed consent before the evaluation.

Out of 154 patients who underwent BAL analysis, 79 fulfilled the selection criteria.

Bronchoalveolar lavage (BAL) was performed as described previously (10). A concentration of $1 \mathrm{x}$ 105 cells/mL was made and a cytospin slide preparation was made (Shandon;Runcorn, UK) and stained with 
May-Grunwald-Giemsa. Two observers blinded to the patients' characteristics counted 400 nonsquamous cells. In accordance with others, active alveolitis was defined as the presence of $\geq 3.0 \%$ polymorphonuclear cells (PMNs) and/or $\geq 2.0 \%$ eosinophils on BAL $(11,12)$. The first aliquot of BAL fluid was used for cultural studies.

Pulmonary Function Tests (PFTs) were performed at baseline and repeated every 12 months. Spirometry was performed according to American Thoracic Society (ATS)/European Respiratory Society (ERS) recommendations (13) and DLCO was measured by a single-breath method, and the results expressed as percent of predicted values based on age, sex and height (14). We considered a value of FVC $<80 \%$ and DCO $<80 \%$ of the predicted value abnormal. During the follow-up we considered worsened patients with a decrease of DLCO >15\%, and of $\mathrm{FVC}>10 \%$.

High-resolution computed tomography (HRCT) scans were scored as reported by Warrick et al (15) independently by 2 experienced observers, who were blinded with regard to clinical and lung function information. We considered a total score value $\geq 8$ abnormal.

${ }^{99 m}$ Technetium-DTPA Lung Scintigraphy. The ${ }^{99 m}$ Tc-DTPA aerosol was produced and administered as described by Coates and Brodovich (16). The normal range for ${ }^{99 m}$ Tc-DTPA-Clearance was defined as a clearance half-time $>40$ $\min$.

Statistical analysis: Data were expressed as the mean \pm standard deviation. Summary statistics were generated using Student's T test or Chi square analysis. Stepwise multiple regression analyses were constructed to determine whether the distribution of BAL count was predictive of outcome or different clinical features of SSc. 


\section{RESULTS:}

Baseline demographics and clinical and serological data for all patients are presented in Table 1. As expected there were more women than men (F:M=8.8:1).

BAL findings indicative of "active alveolitis" were evident in 31 patients: a neutrophilic alveolitis was found in 12; eosinophilic alveolitis in 3, mixed alveolitis (neutrophilic+eosinophilic) in 16 patients. The other subjects presented a negative BAL examination. A significant predominance of anti-Topo1 antibodies was found in the alveolitis group ( $\mathrm{p}=$ $0.017)$ and of ACA antibodies in patients without alveolitis $(\mathrm{p}=0.004)$. No other differences were identified between the two groups with respect to age, subset or duration of SSc, and skin score.

PFTs and HRCT scores are reported in Tables 2 and 3. ACA-positive patients showed significantly lower HRCT scores than patients with anti Topo1-positive antibodies or without both antibodies and only ANA positivity. Alveolar clearance was significantly accelerated in patients with diffuse SSc and in patients with anti-Topo1 antibodies compared to the patients with ACA antibodies (tables 2-3).

Characteristics of pulmonary involvement are reported in Table 4. Compared to patients without alveolitis, those with alveolitis had a significant reduction of DLCO $(\mathrm{p}=0.000), \mathrm{FVC}(\mathrm{p}=0.002)$, more elevated HRCT scores (Total Score $\mathrm{p}=$ 0.000; Severity Score $\mathrm{p}=0.000$; Extent Score $\mathrm{p}=0.000)$ and more accelerated alveolar clearance $(\mathrm{p}=0.020)$. Moreover, the group with alveolitis showed a significantly higher percentage of patients with honeycombing $(\mathrm{p}=0.020)$ but not with ground glass appearance.

Sixty-two patients (26 with pathological and 36 with normal BAL findings), were prospectively followed up for 18-36 months and re-evaluated by clinical examination and lung function tests. 17 patients were missed or excluded due to incomplete data. 
Among 36 patients with normal BAL, 11 (30.5\%) showed stable lung function parameters, 13 improved (36.1\%) and 12 worsened (33.3\%), in this last group 2 patients died of extra-pulmonary complications (the first after 12 months and the second after 18 months from the basal evaluation). In this group, 6 patients with relevant progression of lung fibrosis were treated with cyclophosfamide (CYC) $(750 \mathrm{mg} / \mathrm{m} 2 /$ monthly for 6 months): 3 of them improved and 3 remained stable. The other 6 patients were not treated because they presented reasonable PFT values and clinical conditions.

In the alveolitis group, 12 patients (46.1\%) showed stable lung function parameters and 14 worsened $(53.8 \%) ; 5$ of them died ( 2 for pulmonary fibrosis and related hypertension, 1 from lung cancer, 2 due to heart failure). Twenty patients (77\%) received CYC: $9(45 \%)$ remained stable and $11(55 \%)$ worsened including the 5 patients that died. The remaining 6 patients were not treated for severe co-morbidities; of these patients, 3 remained stable and 3 worsened.

The multiple regression analyses did not show any significant correlation between BAL-positive and BAL-negative patients (data not reported). However, the alveolitis group showed a trend towards a more severe evolution of lung involvement: $33.3 \%$ of non alveolitis patients compared to $53.8 \%$ of the alveoltis group had worsened at the end of the follow up $(\mathrm{p}=0.1)$. 


\section{DISCUSSION}

Interstitial Lung Disease (ILD) frequently complicates SSc, leading to loss of quality of life and poor prognosis. Recognition of interstitial fibrosis in SSc may be delayed due to insidious initial clinical signs and symptoms; moreover, the disease course of fibrosing alveolitis may vary considerably, making treatment decision difficult. With the assumption that alveolitis causes progressive fibrosis, the measurement of inflammatory cells on BAL has become a common clinical practice, useful in identifying patients with ILD who warrant immunosuppression.

Our study revealed a trend towards a more severe course of ILD in the alveolitis group. The absence of significant correlations is probably related to the low number of examined subjects and to a selection bias related to the fact that all the patients already had pulmonary interstitial involvement (see inclusion criteria). Great differences in the prevalence of alveolitis have been reported in past studies, varying from 48 to $72 \%$, probably related to the different ratio of patients with diffuse and limited disease and /or to the different autoantibody subsets (11).

Previous studies underlined the strong correlation between anti-topo-1 antibodies and pulmonary fibrosis (8). Our study confirmed this correlation, showing a more severe HRCT score and a more accelerated alveolar clearance in patients with anti Topo-1 antibodies compared to patients with ACA antibodies. The subgroup of patients without ACA and 
topo- 1 antibodies are more similar to the anti-Topo-1 positive patients than ACA-positive patients, suggesting that the presence of ACA antibodies is related to lower lung involvement and probably with a better prognosis (see table 2).

Moreover, the patients with alveolitis showed a more severe lung disease as indicated by a worse lung function, poorer total, extent and severity HRCT scores and more accelerated alveolar clearance. Alveolar clearance is an index of epithelial permeability and has been used as a marker of alveolar inflammation in a number of pulmonary pathologies $(17,18,19)$. In our experience, in non-smoking patients, alveolar clearance may be a further tool in identifying patients with a higher probability of presenting an active alveolitis.

BAL study has been considered to have a prognostic role in predicting increased mortality and the evolution of FVC and DCO over time. These outcomes also depend on whether the patients were treated with CYC (1) or not. Patients with SSc and ILD without alveolitis had a more stable course while patients with alveolitis showed a significant decline of lung function if not treated.

However, the value of BAL analysis has recently been questioned because it is not yet clear whether a BAL neutrophilia discloses more progressive disease once the severity of disease has been taken into account. Neutrophilia is almost always present when disease is extensive in the lavaged lobe, as judged by HRCT. However, neutrophil levels do not differ in patients with less extensive lobar involvement and in cases with no HRCT abnormalities (20). Moreover, patients with BAL neutrophilia tend to have lower DCO levels (21). Thus, the major influx of neutrophils may occur when disease is already extensive (and intrinsically more progressive), information that can also be readily obtained from HRCT and pulmonary function evaluation. However, BAL neutrophilia seems to be associated to early mortality but not to late mortality (22), underlining the fact that BAL may be clinically inappropriate, at least in patients with severe lung disease. Recent data from Scleroderma Lung Study group showed that HRCT has a higher sensitivity than BAL in identifying lung alveolitis. A possible explanation is that interstitial lung disease begins in the lower lobes and at bronchoscopy BAL tends to be performed in the right middle lobe or lingual: for this reason alveolar disease can be missed (23). In addition, BAL requires experienced personnel to interpret the differential cell count. Therefore, caution is warranted in making treatment decisions solely on BAL findings.

In our analysis, through a follow-up of 12-36 months, we found that alveolitis patients revealed a poor prognosis; most of them worsened or died, even if treated. Moreover, our results are in agreement with data recently reported by two randomized controlled trials (24) showing only a modest beneficial effect of CYC on lung function in SSc patients. These unsatisfying results could also be related to the advanced-stage of the disease in our cohort of patients. In fact, a large number of our patients with alveolitis presented signs of irreversible fibrosis on HRCT.

In conclusion, BAL remains the only tool to exclude lung infections and other causes of lung abnormalities. In our opinion, due to an evident overlap in HRCT scores between patients with or without alveolitis, in the single case the 
evaluation of only HRCT and/or PFTs may not be sufficient as prognostic tools, and BAL analysis can be considered a useful instrument in evaluating SSc patients with ILD.

\section{Disclosures}

I would like to make the following statements:

1) All authors have contributed significantly to the research described in the paper and have read and approved the final manuscript

2) the manuscript is the original work of the authors

3) neither this manuscript nor another manuscript with substantially similar content under our authorship has been published, has been accepted, or is being considered elsewhere for publication

4) there are no affiliations or financial involvement with any entity with a financial interest in the subject matter. No conflict of interest.

5) all individuals who have contributed significantly to the work reported in this manuscript are acknowledged by name and have granted permission to be named.

The authors declare that they have no conflict of interest:

Stefania Volpinari: none

Renato La Corte: none

Stefano Bighi: none

Franco Ravenna: none 
Napoleone Prandini: none

Andrea Lo Monaco: none

Francesco Trotta: none.

\section{References}

1. Steen VD, Conte C, Owens GR, Medsger TA Jr (1994) Severe restrictive lung disease in systemic sclerosis. Arthritis Rheum 37:1283-1289 Medline. doi:10.1002/art.1780370903

2. White B, Moore WC, Wigley FM, Xiao HQ, Wise RA (2000) Cyclophosphamide is associated with pulmonary function and survival benefit in patients with scleroderma and alveolitis. Ann Intern Med 132:947-954 Medline.

3. Baker AM, Bowton DL, Haponik EF (1995) Decision making in nosocomial pneumonia. An analytic approach to the interpretation of quantitative bronchoscopic cultures. Chest 107:85-95 Medline. doi:10.1378/chest.107.1.85

4. Strange C, Bolster MB, Roth MD, et al (2008) Bronchoalveolar lavage and response to cyclophosphamide in scleroderma interstitial lung disease. Am J Respir Crit Care Med 177:9198 Medline. doi:10.1164/rccm.200705-6550C

5. Subcommittee for Progressive Systemic Sclerosis (1980) Criteria of the American Rheumatism Association Diagnostic and Therapeutic Criteria Committee: Preliminary Criteria for the Classification of Systemic Sclerosis (Scleroderma). Arthritis Rheum 23:581-590 Medline. doi:10.1002/art.1780230510

6. LeRoy EC, Black C, Fleischmajer et al. (1988) Scleroderma (Systemic sclerosis): Classification, subset and pathogenesis. J Rheumatol 15:202-205 Medline. 
7. Kahaleh MB, Sultan GL, Smith EA, et al (1986) A Modified scleroderma skin scoring method. Clin Exp Rheumatol 4:367-369 Medline.

8. Sato S, Hamaguchi Y, Hasegawa M, Takeara K (2001) Clinical significance of antitopoisomerase I antibody levels determined by ELISA in systemic sclerosis. Rheumatology 40:1135-1140 Medline. doi:10.1093/rheumatology/40.10.1135

9. Mahler DA, Weinberg DH, Wells CK, Feinstein AR (1984) The measurement of dyspnea: contents, interobserver agreement, and physiologic correlates of two new clinical indexes. Chest 85:751-758 Medline. doi:10.1378/chest.85.6.751

10. Klech H, Pohl W (1989) Technical recommendations and guidelines for bronchoalveolar lavage (BAL). Report of the European Society of Pneumology Task Group on BAL. Eur Respir J 2:561-585 Medline.

11. Behr J, Vogelmeier C, Beinert T, et al (1996) Bronchoalveolar lavage for evaluation and management of scleroderma disease of the lung. Am J Respir Crit Care Med 154:400-406 Medline.

(12) The Joint statement of the ATS and ETS: Idiopathic pulmonary fibrosis: diagnosis and treatment, International Consensus Statement. (2000) Am J Respir Crit Care Med 161: 646-64.

13. American Thoracic Society (1995) Standardization of spirometry-1994 update. Am J Respir Crit Care Med 152:1107-1136 Medline.

14. American Thoracic Society: Single breath carbon monoxide diffusing capacity (transfer factor). Recommendation for a standard technique (1995) Update (1995). Am J Respir Crit Care Med 152:2185-2198 Medline.

15. Warrick JH, Balla M, Schabel SI, Silver RM (1991) High resolution computer tomography in early scleroderma lung disease. J Rheumatol 18:1520-1528 Medline.

16. Coates G, O’Brodovich H (1986) Measurement of pulmonary epithelial permeability with Tc99m-DTPA aerosol. Semin Nucl Med 16:275-284 Medline. doi:10.1016/S00012998(86)80014-9

17. Lo Monaco A, La Corte R, Prandini N, et al (2005) Alveolar Clearance of 99mTc-DTPA, Pulmonary high resolutioon CT and respiratory function tests correlations in the evaluation of lung involvement in 207 patients with Systemic Sclerosis. Arthritis Rheum 52(suppl):S163.

18. Kon OM, Danil Z, Black CM, du Bois RM (1999) Clearance of inhaled Tc-99m-DTPA as a clinical index of pulmonary vascular disease in systemic sclerosis. Eur Respir J 13:133-136 Medline. doi:10.1183/09031936.99.13113399

19. Diot E, Giraudeau B, Maillot F, Diot P (1999) Decrease in DLCO in systemic sclerosis correlates with acceleration of DTPA clearance. Eur Respir J 14:728-729 Medline. doi:10.1034/j.1399-3003.1999.14c42.x

20. Wells AU, Hansell DM, Rubens MB, et al (1994) Fibrosing alveolitis in systemic sclerosis: bronchoalveolar lavage findings in relation to computed tomographic appearances. Am J Respir Crit Care Med 150:462-468 Medline.

21. Wells AU, Hansell DM, Haslam PL, et al (1998) Bronchoalveolar lavage cellularity: lone cryptogenic fibrosing alveolitis compared with the fibrosing alveolitis of systemic sclerosis. Am J Respir Crit Care Med 157:1474-1482 Medline.

22. Goh NSL, Veeraraghavan S, Desai SR, et al (2007) Bronchoalveolar lavage and cellular profiles in patients with Systemic Sclerosis-Associated Interstitial Lung Disease are not predictive of disease progression. Arthritis Rheum 56:2005-2012 Medline. doi:10.1002/art.22696 
23. Clements PJ, Goldin JG, Kleerup EC, et al (2004) Regional differences in bronchoalveolar lavage and thoracic high-resolution computed tomography results in dyspneic patients with Systemic Sclerosis. Arthritis Rheum 50:1909-1917 Medline. doi:10.1002/art.20265

24. Fathi N, Furst DE, Clements PJ (2007) Management of Interstitial Lung Disease in Systemic Sclerosis: Lessons from SLS and FAST. Curr Rheumatol Rep 9:144-150 Medline. doi:10.1007/s11926-007-0009-y 